\title{
EL APRENDIZAJE DEL CÁLCULO DIFERENCIAL MEDIANTE EL USO DE UN SOFTWARE MULTIMEDIA
}

\author{
RAÚL FERNÁNDEZ AEDO \\ RAMÓN CABRERA REYES \\ UNIVERSIDAD DE CIEGO DE ÁVILA \\ CENTRO DE ESTUDIO DE GESTIÓN DE \\ LA INFORMACIÓN Y EL CONOCIMIENTO
}

CUBA

"Saber cómo utilizar tecnología no es lo mismo que saber cómo enseñar con tecnología". Punya Mishra - Matthew Koehler. 


\title{
RESUMEN
}

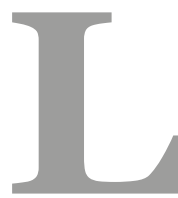

as herramientas TIC juegan en la sociedad un papel clave que permite logros antes ni soñados. La enseñanza de la Matemática y en especial el tema del cálculo diferencial es de uso diario en las carreras de ingeniería o en aquellas que tocan aspectos de estos pudiéndose corroborar que es uno de los temas que más dudas y problemas trae a los futuros profesionales. El aprendizaje, con el uso de las tecnologías multimedias mejoran la calidad de la enseñanza. El uso de ella permite el autoaprendizaje por parte de los estudiantes y además se emplean métodos que permitan reforzar el conocimiento de estos.

Palabras Claves: cálculo diferencial, multimedia, autoaprendizaje.

\begin{abstract}
The tools TIC play in the society a key paper that allows achievements before neither dreamt. The teaching of the Mathematical one and especially the topic of the differential calculation is of use newspaper in the engineering careers or in those that play aspects of these being able to corroborate that it is one of the topics that more doubts and problems bring to the professional futures. The learning, with the use of the technologies multimedias improves the quality of the teaching. The use of her allows the autoaprendizaje on the part of the students and methods are also used that allow to reinforce the knowledge of these.
\end{abstract}

Key words: differential calculation, multimedia, autoaprendizaje.

\section{DESARROLLO}

Las tendencias actuales en la enseñanza identifican los avances tecnológicos como un valioso recurso capaz de acompañar el aprendizaje de distintas materias en cualquier etapa educativa. En este sentido, Cuba ha identificado desde muy temprano la conveniencia y necesidad de dominar e introducir en la práctica social las Tecnologías de la Información y las Comunicaciones y lograr una cultura digital como una de las características imprescindibles del hombre nuevo, lo que facilitaría a nuestra sociedad acercarse más hacia el objetivo de un desarrollo sostenible.

Según Area (2002): "Multimedia" significa la conjugación de diferentes medios de comunicación. Teniendo en cuenta esta definición se ha reconocido a la Multimedia como la integración de dos o más medios de comunicación que pueden ser controlados, manipulados por el usuario en una computadora, es decir, es un sistema informático interactivo, controlable por el usuario, que integran diferentes medios como el texto, video, imagen, sonido y las animaciones, y capaz de combinar los medios de comunicación y el Hombre-Máquina. 
En la actualidad existen varios software educativos en la rama de las matemáticas incluso en el tema que nos ocupa pero la mayoría son dirigidos fundamentalmente al cálculo y no a la enseñanza de los conocimientos que deben adquirir los estudiantes en la materia y los que realmente tratan el tema para que el alumno se apropie de estos conocimientos no muestran una gran variedad de ejemplos. Según Fernández (2004) uno de los software que existen que trata el tema Cálculo Diferencial es el DERIVE, el cual es muy bueno haciendo cálculo de todo tipo y es capaz de calcular cualquier derivada pero no de enseñarle al estudiante la vía de cómo hacerlo ni de interpretar ningún problema que conduzca a la aplicación de la derivada, ni tiene ninguna información teórica al respecto, ni ejemplos donde el estudiante vea el procedimiento que se aplicó para su solución.

Con nuestro trabajo precisamente lo que pretendemos es enseñar al estudiante a razonar profundamente situaciones que conllevan a la aplicación del cálculo diferencial, enseñarlo a derivar, mostrarle información que pueda consultar para profundizar en sus conocimientos o aclarar cualquier duda, mostrarle muchos ejemplos de todo tipo incluyendo de aplicación así como una guía de ejercicios para la ejercitación y consolidación de los conocimientos, además de varios temarios de exámenes donde se puede autoevaluar, ya que el estudiante deberá resolver las preguntas y seleccionar la respuesta correcta y obtendrá una calificación.

Para la realización de dicha multimedia se empleo la metodología Multimet de Alvarez (2004) y para su programación el lenguaje de autor Mediator 8.

La Multimedia educativa DERIVADAS comienza con la pantalla siguiente:

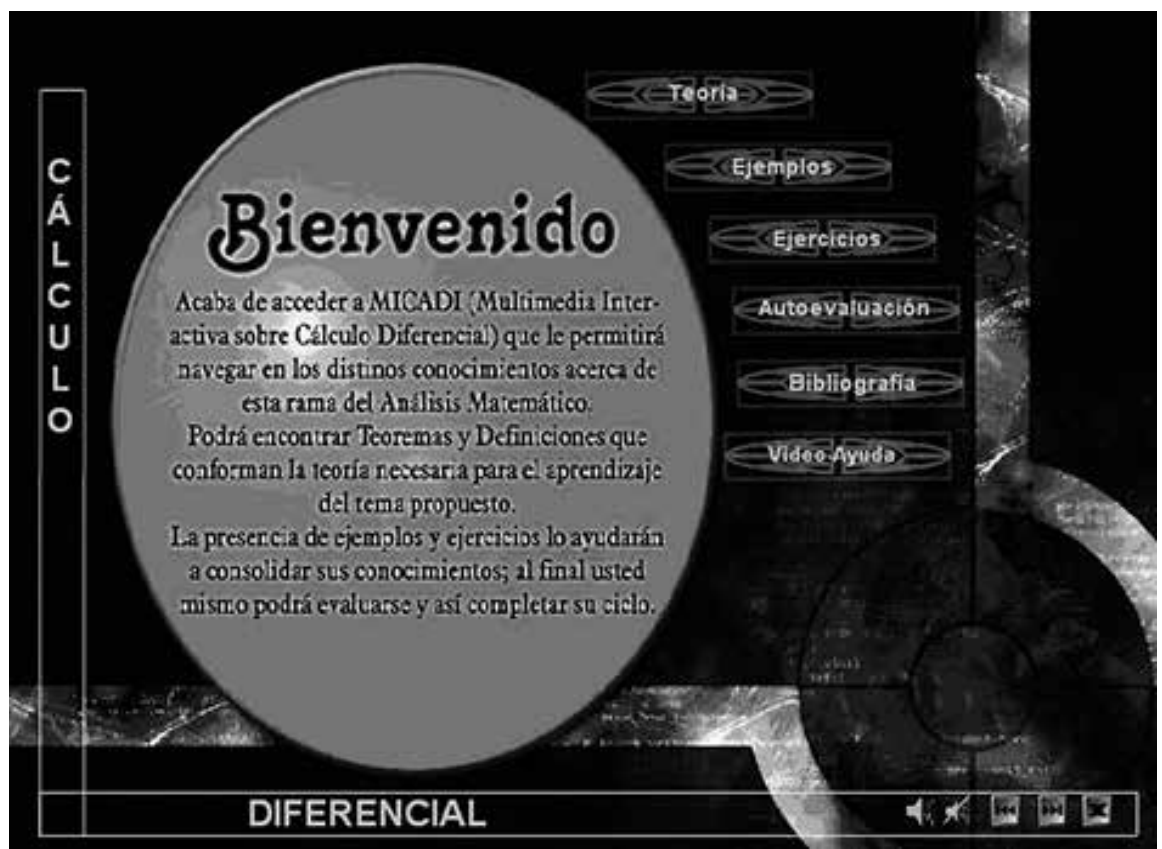

Figura 1: Pantalla Inicial 
Como se puede observar en la misma aparece un menú de opciones que permiten al estudiante ir navegando hacia los temas que desee.

La opción teoría muestra información acerca de la teoría existente en cuanto al tema:
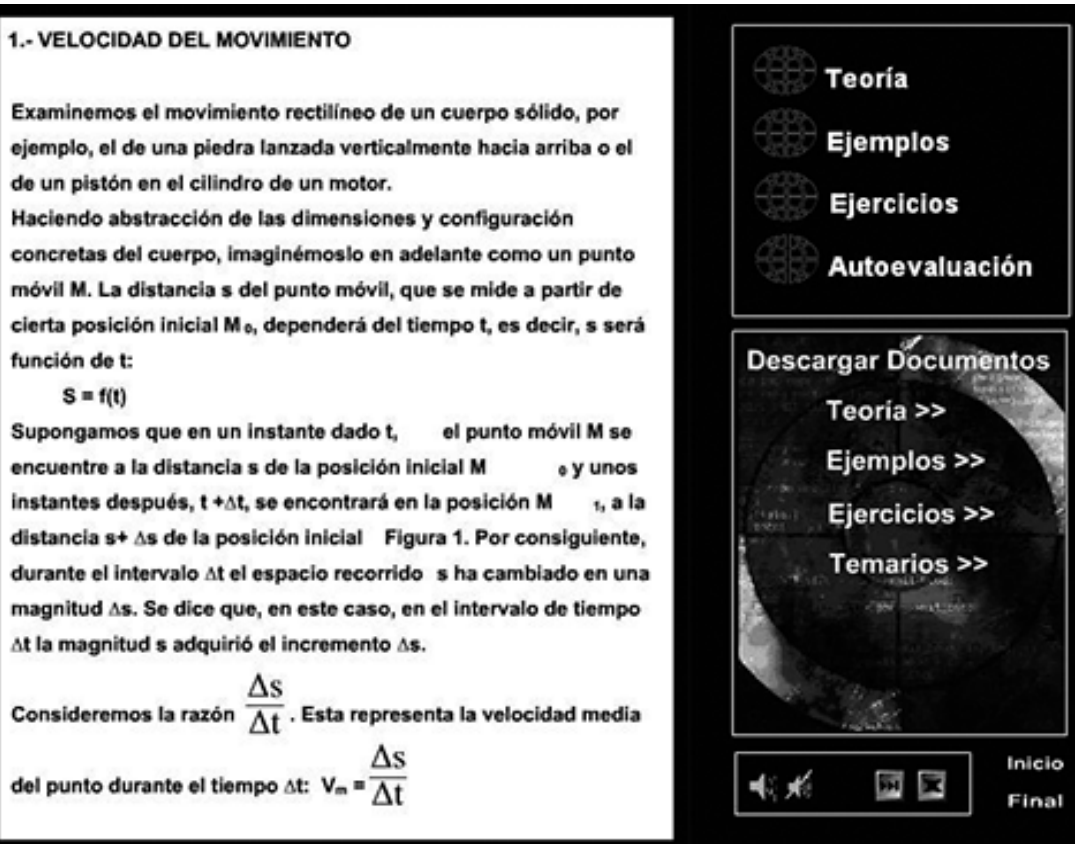

Figura 2: Teoría

La opción ejemplos muestra ejemplos resueltos del tema:

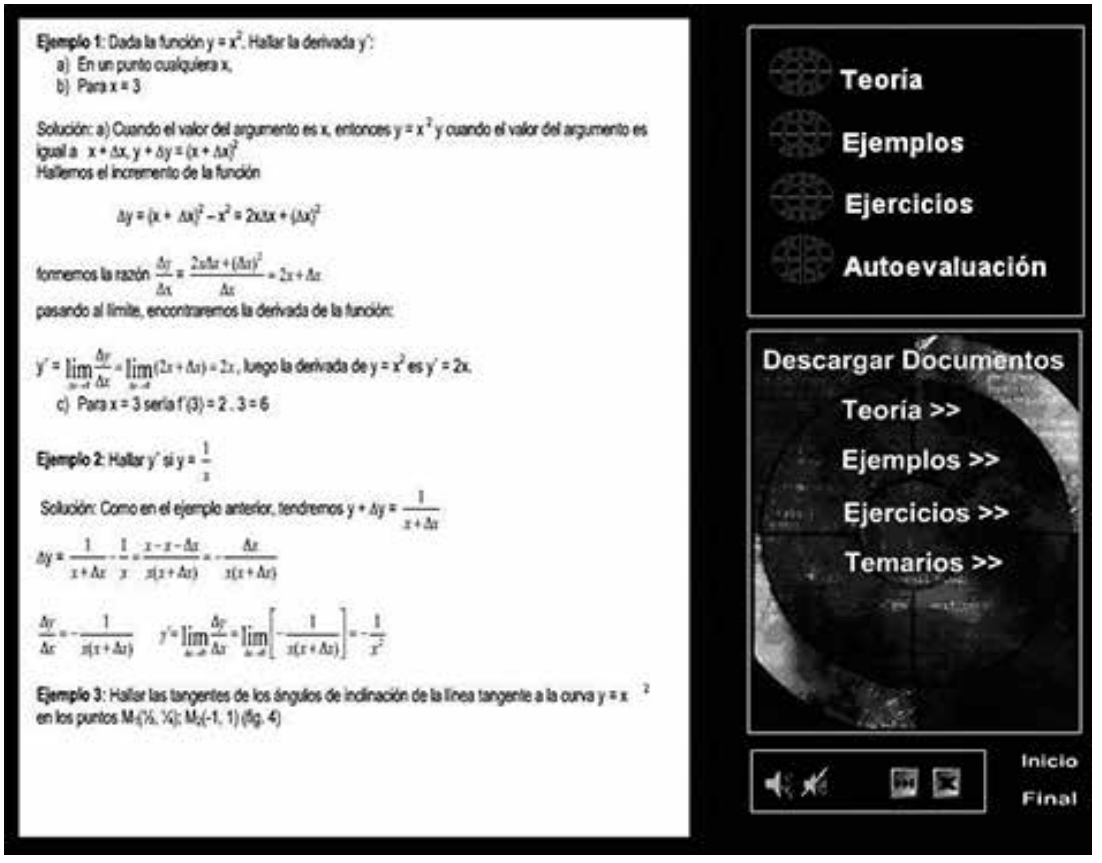

Figura 3: Ejemplos 
La opción ejercicios muestra una serie de ejercicios propuesto para que el alumno pueda resolverlos y comprobar su solución, muchos de los mismos fueron tomados de autores como Ayres (1988) y Demidovich (1990):

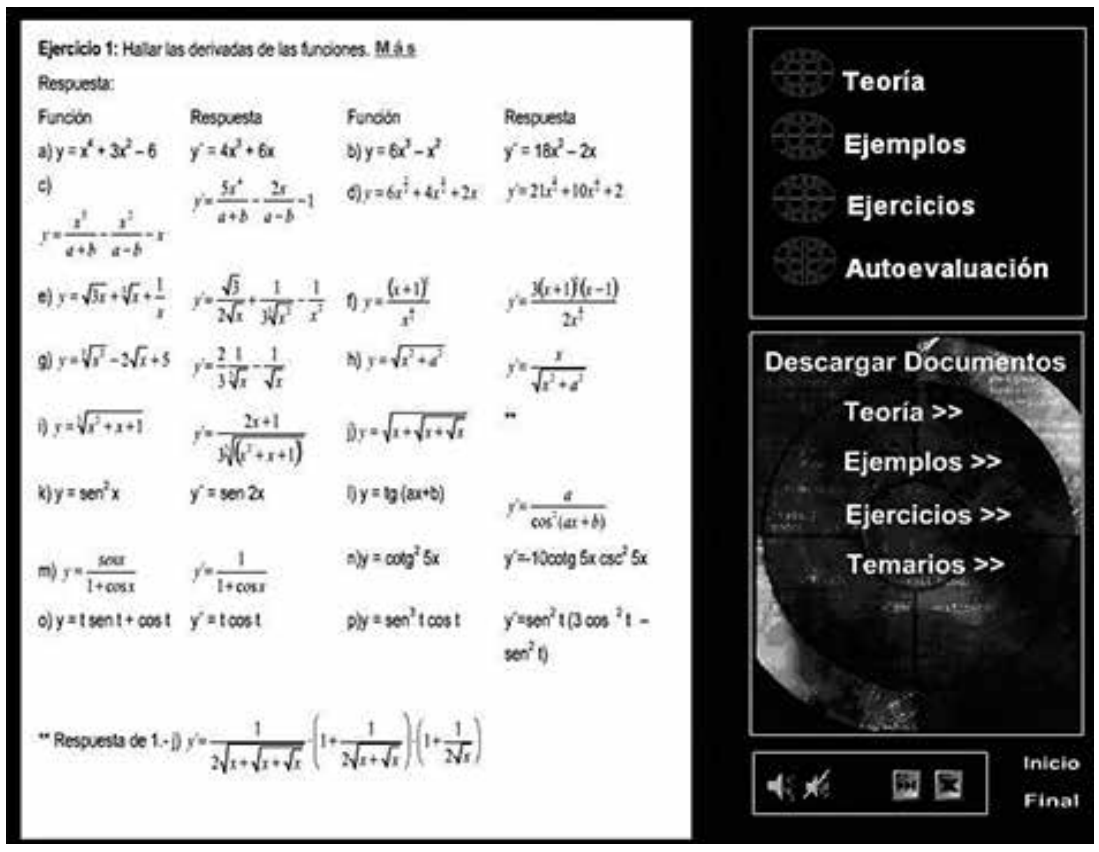

Figura 4: Ejercicios

La opción bibliografía le da al alumno una serie de sugerencias de donde encontrar el contenido tratado.

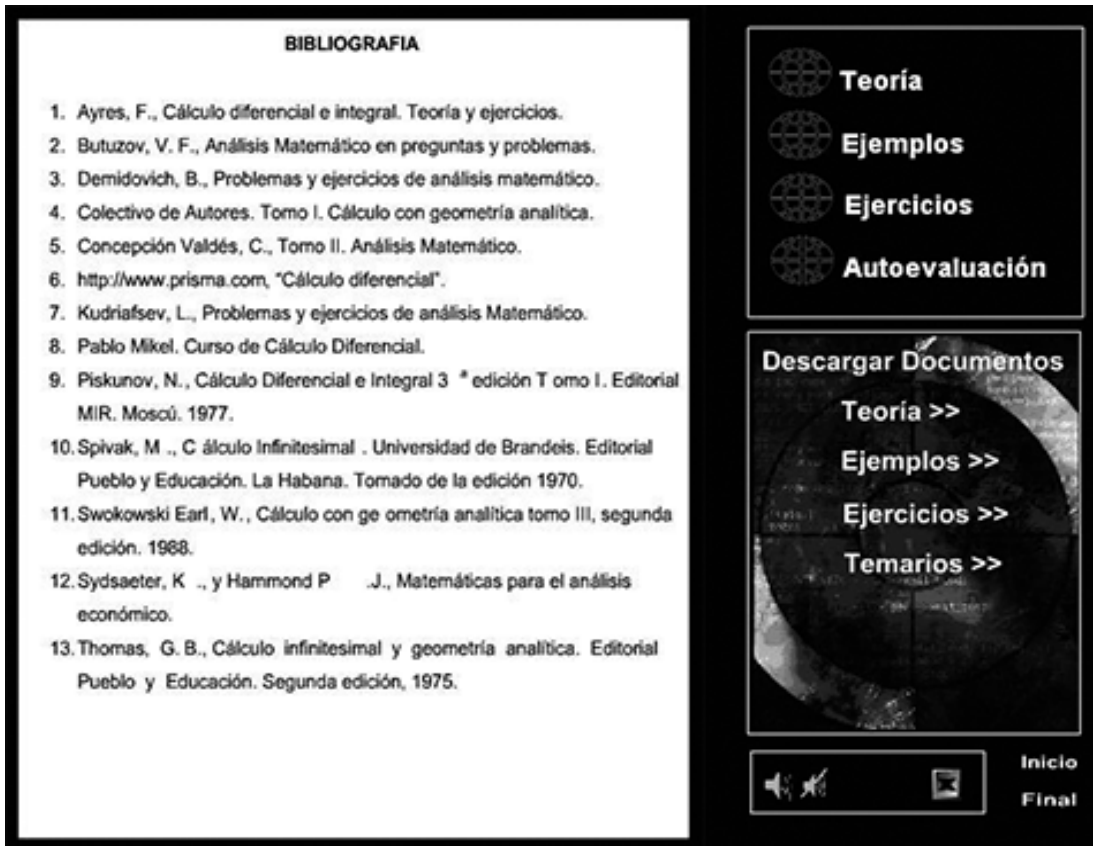

Figura 5: Bibliografía 
Por último la autoevaluación que son una serie de ejercicios que aparecen aleatoriamente que permiten al estudiante autoevaluar sus conocimientos. El software además trae un video explicativo de cómo realizar la autoevaluación así como una ayuda acerca del funcionamiento del mismo.

Los resultados fueron corroborados mediante criterio de experto (Fernández, 2005) para lo cual se seleccionaron 19 expertos mediante el uso del coeficiente de competencia (k) y se valoró la eficacia del producto mediante el método de las preferencias el cuál revelo la concordancia de estos en cuanto a la efectividad de la multimedia tanto en su concepción teórica como en los resultados que se obtendrán con su aplicación. Es un software que permite el aprendizaje de los temas de Cálculo Diferencial de forma amena y sencilla además es una eficiente herramienta para la consolidación de estos contenidos además que puede ser utilizado por todos los estudiantes que en su programa de estudio traten el tema de las derivadas.

\section{CONCLUSIONES}

La multimedia para la enseñanza del cálculo diferencial está diseñada para su fácil manipulación, con ambiente agradable y hace que el estudiante se interese por la utilización y explotación del mismo para la adquisición de los conocimientos en el tema Cálculo Diferencial. Es una herramienta que sirve como material complementario y medio de enseñanza del tema en las diferentes carreras que lo enseñen. Permite el autoaprendizaje al vincular todos los sentidos en su diseño.

\section{REFERENCIAS BIBLIOGRAFICAS}

Area, M. "Problemas y retos educativos ante las tecnologías digitales en la sociedad de la información". En Revista Quaderns Digitals, nº 28. 2002.

Alvarez, S. "Metodología Multimed para el desarrollo de aplicaciones multimedias”. ISJAE. Cuba. 2004.

Ayres, F. Cálculo diferencial e integral. Teoría y ejercicios. Cuba. 1988.

Demidovich, B. Problemas y ejercicios de análisis matemático. Editorial Mir. Moscú. 1990.

Fernández, Aedo, R. “Folleto para la evaluación de investigaciones”. UNICA. 2005. 


\section{CURRICULUM VITAE}

Fernández Aedo, Raúl

Graduado de Licenciatura en Educación especialidad Matemática, Master en Computación Aplicada y Doctor en Ciencias Pedagógicas. Posee más de 30 publicaciones en revistas nacionales e internacionales. Es autor de varios libros publicados en Cuba, Argentina y España. Sus líneas principales de investigación son la Enseñanza a Distancia, Informática Educativa y Tecnología Educativa. Ha participado en más de 40 eventos nacionales e internacionales. Es jefe de un grupo de investigación en Tecnología Educativa.

aedo@informatica.unica.cu

\section{Cabrera Reyes, Ramón}

Graduado de licenciatura en Educación especialidad Matemática. Master en Nuevas Tecnologías para la Educación. Profesor de Matemática de la Sede Universitaria. Ha participado en eventos nacionales como el COMPUMAT, evento UNICA y FORUM de Ciencia y Técnica. Posee varias publicaciones.

aedo@informatica.unica.cu 